\title{
Tale of Two Alkaloids: pH-Controlled Electrochemiluminescence for Differentiation of Structurally Similar Compounds
}

\author{
Kelly Brown, Charlotte Jacquet, Julien Biscay, Pamela Allan, and Lynn Dennany*(e) \\ WestChem Department of Pure and Applied Chemistry, University of Strathclyde, Technology and Innovation Centre, 99 George \\ Street, Glasgow G1 1RD, U.K.
}

Supporting Information

\begin{abstract}
Electrochemiluminescence (ECL) has increased in popularity as a result of its inherent advantages, including but not limited to portability, simplicity of use, and low reagent consumption. However, its significant advantages are often over shadowed as a result of its limited specificity. ECL emissions are intrinsically broad and lack the definition of other available analytical techniques. Furthermore, species with similar functional groups have almost identical electrochemical behavior and thus typically emit within approximately the same potential region. Within this contribution we have demonstrate the use of $\mathrm{pH}$ controlled ECL to prove the presence of two individual species within a mixed sample. Analysis at a single $\mathrm{pH}$ would not provide

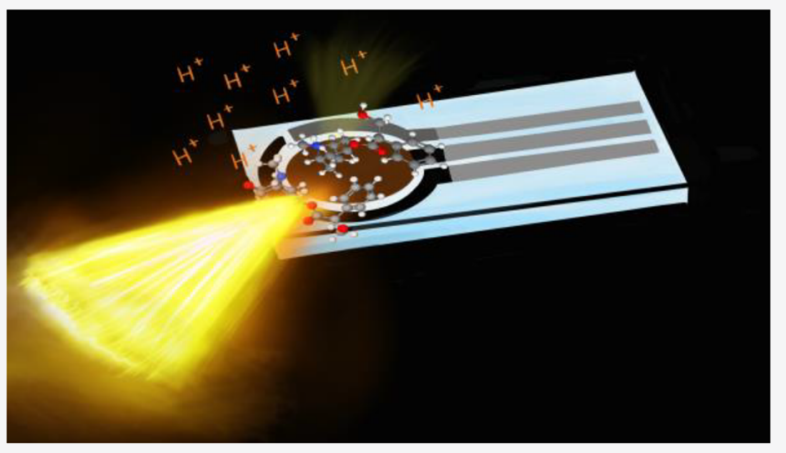
this information. We have illustrated the potential of this methodology to quantify scopolamine alongside sister tropane alkaloid atropine, a known ECL interferent. Previously the two alkaloids could not be distinguished from one another using a single technique which did not involve a separation strategy. $\mathrm{pH}$ controlled ECL is a simple approach to improve the specificity of a basic $\left[\mathrm{Ru}(\mathrm{bpy})_{3}\right]^{2+}$ film based sensor. By exploiting molecular characteristics, such as $\mathrm{p} K_{\mathrm{a}}$, we have been able to fine-tune our methodology to facilitate identification of analytes previously exhibiting indistinguishable ECL emission. Thus, by improving specificity, while maintaining operational simplicity and inexpensive design, we have been able to highlight the potential power of ECL for identification of structurally similar compounds. Further improvements of specificity, such as demonstrated within this contribution, will only further future applications of ECL sensors across a range of different fields.
\end{abstract}

$\mathrm{T}$ he ability to identify compounds with confidence is a fundamental concept of analytical chemistry. Often analysis of samples, regardless of the field, requires the detection of compounds based upon their structural properties. This can range from impurity analysis in pharmaceuticals to identification of illicit substances within a forensic context. As analytical chemistry moves toward greater portability, the requirement to simplify instrumentation, minimize cost, and decrease reagent consumption is becoming a key driving force within method development. ${ }^{1-3}$ Electrochemiluminescence (ECL) satisfies a number of these criteria. ${ }^{4-7}$ By combining the advantages of electrochemistry with photoluminescence, ECL stands out from other analytical techniques. Unlike its counterparts, ECL produces luminescence without the requirement of an external light source, vastly simplifying the instrumentation required, allowing it to be packaged within lightweight portable systems. ${ }^{4-8}$ Furthermore, the recent advances in both electrochemical (EC) and ECL systems have demonstrated the ability to negate the need for potentiostats by utilizing mobile phones to apply the required voltage alongside the use of their in-built cameras as the detector, thus producing low cost, portable, and operationally simple devices. ${ }^{9,10}$
As the technology to perform ECL progresses, one significant limitation remains. Specificity has long plagued both EC and ECL techniques. The principles upon which both techniques operate suffer from a lack of specificity as a result of the redox chemistry across functional groups remaining largely similar. ${ }^{5}$ One commonly encountered functional group which suffers from this lack of specificity are amines. A large number of amine compounds have been shown to be strong ECL coreactants. However, their redox chemistry and hence emission mechanisms are largely similar, and thus their signals are observed across the same potential range. ${ }^{5,11-15}$ This can make identification of a specific species problematic and can limit identification to a high level functional group classification. Furthermore, similar species such as tropane alkaloids, which contain a tertiary nitrogen group within their tropane ring, are also observed to emit in this region and thus further complicate class identification. ${ }^{16-18}$ Therefore, it has been necessary until now to employ separation strategies, such as chromatography or capillary electrophoresis (CE) prior to

Received: October 29, 2019

Accepted: December 23, 2019

Published: December 23, 2019 
EC or ECL detection such that analytes which are structurally similar are identified via their retention or migration times. $^{19-22}$

A number of methodologies have been recently established to improve the specificity of EC and ECL techniques. These largely focus on the addition of a secondary species to modify the electrode surface. Commonly employed methods include the addition of biological molecules such as single stranded apatmers $^{23-25}$ and antibodies ${ }^{8,26}$ but can also include nonbiological materials such as Schiff bases, ${ }^{27,28}$ carbon nanotubes, $^{29}$ fluorescent probes $^{30}$ and specifically designed metal complexes. ${ }^{31,32}$ Biological species in particular demonstrate a high degree of selectivity toward the molecule of interest. These can be specifically designed to interact with one particular species. Following this interaction, a "signal off" or "signal on" approach is observed. When the target analyte is present, the ECL probes are either released from the electrode surface, thus a decrease in ECL is observed or are brought close to the electrode surface thus facilitating the commencement of electrochemistry, resulting in the increase or appearance of a signal. ${ }^{23,25}$ Alternative biological based approaches investigate the incorporation of the recognition group into the coreactant species rather than the electrode surface. This technique showed promise and offered a simpler methodology than electrode modification previously proposed. $^{33}$

Although the above mention techniques have demonstrated a high degree of specificity, making them powerful modification strategies, they are both complex and expensive to perform. In contrast, we propose within this contribution a simple strategy to increase ECL specificity without requiring the inclusion of additional species to the electrode surface, bar the necessary luminophore. Despite their almost identical chemical structure and electrochemical properties, we have been able to differentiate between two tropane alkaloids, atropine sulfate and scopolamine hydrobromide. By effectively switching off emission from atropine, while maintaining scopolamine emission, we have been able to quantify scopolamine in the presence of atropine. Exploiting the difference in $\mathrm{p} K_{\mathrm{a}}$ values and subsequently adjusting sample $\mathrm{pH}$ accordingly, we have shown that emission from a basic $\left[\mathrm{Ru}(\mathrm{bpy})_{3}\right]^{2+}$ ECL sensor can be tuned to provide greater specificity. Furthermore, unlike the biological or chemical modifications, which require expertise to perform, our simple system could be easily adapted to accommodate a range of skills levels across a number of fields, thus further widening its applications. In addition, by preventing the use of the selective reagents, we can apply the developed sensor to a wide range of different species without requiring the redesign of electrode modification. By negating the requirement for a separation strategy prior to detection, we have maintained instrument and operational simplicity in addition to portability, all of which will only stand to further encourage the use of basic ECL sensors. Furthermore, expansion of $\mathrm{pH}$ controlled ECL to a more extensive range of compound classes will only stand to further enhance the possible applications of this combination of techniques, thus, illustrating the potential of such a strategy to the wider analytical community.

\section{EXPERIMENTAL SECTION}

Materials. Tris (2,2'-bipyridyl)-dichlororuthenium(II) hexahydrate $\left(\left[\mathrm{Ru}(\mathrm{bpy})_{3}\right] \cdot \mathrm{Cl}_{2} \cdot 6 \mathrm{H}_{2} \mathrm{O}\right)$, atropine sulfate monohydrate, (-)-scopolamine hydrobromide trihydrate (Sc-HBr), lithium perchlorate $\left(\mathrm{LiClO}_{4}\right)$, and 117 Nafion ( 5\% mixture of lower aliphatic alcohols and water) were purchased from Sigma-Aldrich. Absolute EtOH was purchased from VWR Chemicals. All chemicals were used as received. All solutions were prepared in Milli-Q water $\left(18 \mathrm{~m} \Omega \mathrm{cm}^{-1}\right)$.

Instrumentation. A CH instrument model $760 \mathrm{D}$ electrochemical analyzer and Hamamatsu H10723-20 photomultiplier tube (PMT) were used for all electrochemical and photoluminescence measurements. GSI Technologies Electrochemical carbon screen printed electrodes (SPE) with a $4 \mathrm{~mm}$ carbon working electrode, carbon counter, and Ag reference were used throughout. Photoluminescence measurements were performed in a light tight Faraday cage using a specifically designed sensor holder to position the PMT directly above the working electrode.

Fabrication of $\left[\mathrm{Ru}(\mathrm{bpy})_{3}\right]^{2+} /$ Nafion ECL Sensor. The sensor was fabricated following the same procedures previously reported in Brown et al. ${ }^{18}$ Briefly, $7 \mu \mathrm{L}$ of a $1: 1(\mathrm{v} / \mathrm{v})$ of 0.5 $\mathrm{mM}\left[\mathrm{Ru}(\mathrm{bpy})_{3}\right]^{2+}$ and $0.2 \%$ nafion in $50: 50 \mathrm{EtOH} / \mathrm{H}_{2} \mathrm{O}$ was drop-cast onto the electrode surface and allowed to completely air-dry in darkness prior to use. Prior to sample measurements, electrodes were preconditioned by performing three subsequent $\mathrm{CV}$ over the potential range of interest within the electrolyte solution to achieve a stable signal.

\section{RESULTS AND DISCUSSION}

ECL Detection of Atropine and Scopolamine. The ability of a simple ruthenium film-based ECL sensor has been previously shown to reliably detect tropane alkaloids atropine sulfate and scopolamine hydrobromide both within ideal and complex matrixes without the requirement for any sample preparation. $^{18}$ One of the major limitations of single luminophore ECL analysis is the lack of specificity offered as a result of the broad spectral response observed. Species with similar functionalities display similar redox behavior, and as a result, they often produce emission at similar potentials. ${ }^{5}$ Small structural differences have however been seen to demonstrate slight differences in redox potentials. This becomes apparent when examining atropine and scopolamine. The two tropane alkaloids possess almost identical chemical structures, as shown in Figure 1a. However, CV and subsequent ECL analysis of both species individually have shown that the potential at which oxidation occurs differs for each analyte. The CVs for both are shown in Figure $S 1$ with atropine observed at a slightly earlier potential of $\sim 0.89 \mathrm{~V}$ compared with $\sim 1.30 \mathrm{~V}$ observed for scopolamine at unmodified carbon electrodes. The ECL observed for both via their individual interaction with the $\mathrm{Ru}^{2+/ 3+}$ redox couple is observed at $\sim 0.83$ and $0.89 \mathrm{~V}$ for atropine and scopolamine, respectively. This mediated oxidation process is consistent with reported ECL mechanistic pathways for alkaloids ${ }^{17,18,34,35}$ It is believed that these slight differences arise due to the influence of the additional epoxide group present within scopolamines structure. Quantum calculations performed on both species have shown that the $E_{\text {HOMO }}$ for atropine $\left(-9.590 \mathrm{eV}^{36}\right)$ is slightly higher than that observed for scopolamine $(-9.780 \mathrm{eV}) .{ }^{36}$ Furthermore, Màrquez et al. ${ }^{37}$ also reports that $E_{\text {HOMO }}$ for scopolamine exists at lower energies than other alkaloid species including cocaine and tropane. ${ }^{37}$ With the distance between the epoxide and the nitrogen of the tropane group only $2.47 \AA{ }^{38}$ it is proposed that redistribution of the electron density could result in an inductive effect toward the positive nitrogen within the tropane ring system, thus stabilizing the cation. As such the 

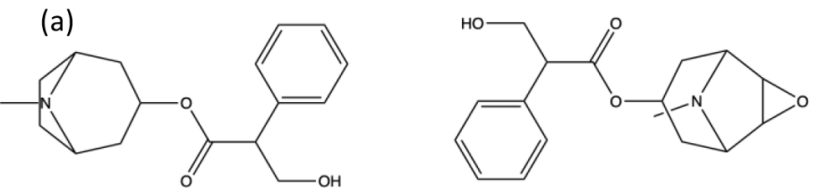

Atropine

Scopolamine
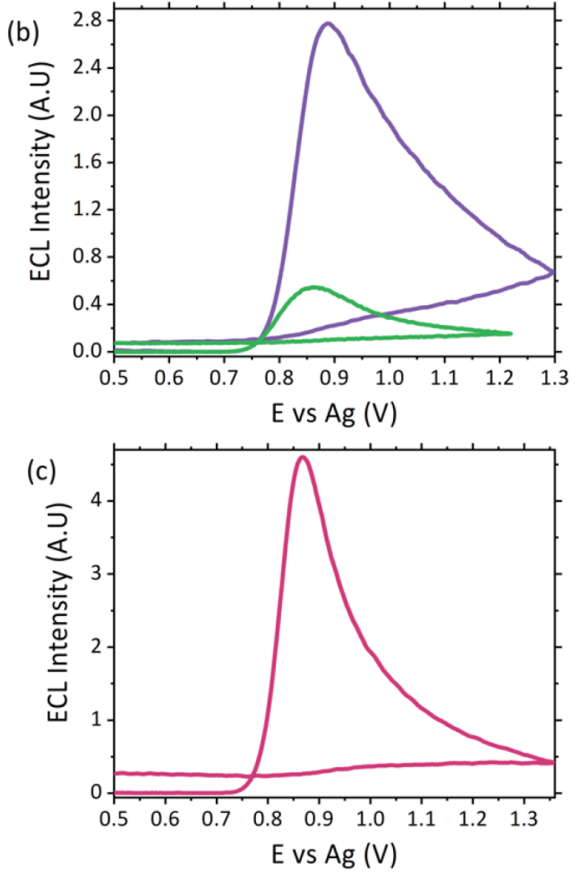

Figure 1. (a) Structures of tropane alkaloids atropine and scopolamine (b) ECL responses of atropine sulfate (green) and scopolamine hydrobromide (purple) with ruthenium modified carbon electrodes at a scan rate of $100 \mathrm{mV} \mathrm{s}^{-1}$ in $\mathrm{pH} 80.1 \mathrm{M} \mathrm{LiClO}_{4}$ electrolyte across 0.5 $\leq E \leq 1.3 \mathrm{~V}$ vs Ag at a PMT setting of $0.6 \mathrm{~V}$. (c) ECL response of mixed atropine sulfate and scopolamine hydrobromide sample in $\mathrm{pH}$ $8 \mathrm{LiClO}_{4}$ with the same parameters used for part b.

HOMO for scopolamine will be observed at a lower energy than atropine, which has no additional electron donating groups present and thus requires the application of more positive potentials to promote electro-oxidation. ${ }^{36-38} \mathrm{~A}$ similar trend has been previously reported for similar species including amino acids where the presence of different functional groups was seen to influence the oxidation potentials as a result of the difference in $E_{\text {HOMO. }}{ }^{11,39,40}$ This slight potential difference is also observed in the ECL intensity (A.U. arbitrary units) of the alkaloids, see Figure $1 \mathrm{~b}$, with atropine's $\lambda_{\mathrm{ECLmax}}$ observed at $\sim 0.83 \mathrm{~V}$ and scopolamine at $\sim 0.89 \mathrm{~V}$. The low onset of ECL has previously been observed for atropine ${ }^{18}$ and can be attributed to the formation of a radical similar to mechanisms outlined previously. ${ }^{41,42}$ Despite the different oxidation potentials of atropine and scopolamine, when a mixed sample is analyzed, the two individual peaks cannot be identified, as shown in Figure 1c. This is not unusual since the broad spectral response observed as a consequence of ECL emission does not offer the distinctive peaks observed in other techniques. Moreover, the oxidative-reduction ECL mechanism which is responsible for their ECL emission is almost identical, since both compounds contain the same tropane alkaloid functionality, responsible for this electrochemical behavior. Furthermore, they also follow the same electrolytic
N-dealkylation degradation pathways, similar to those observed for other structurally similar amine species. ${ }^{18,34,43-46}$ As such, the ability to differentiate between these structurally similar species in addition to other structurally similar amine species utilizing single luminophore ECL alone is extremely challenging.

$\mathrm{pH}$ Impact on ECL Intensity. It is well-known that $\mathrm{pH}$ significantly influences the intensity of ECL emission, as a result of whether the oxidizable form of the analyte is dominate at a particular $\mathrm{pH}$. As such, the greatest intensities are observed close to the $\mathrm{p} K_{\mathrm{a}}$ value of the species. ${ }^{14,17,18,34,47} \mathrm{pH}$ analysis of atropine previously ${ }^{18}$ has shown negligible emission is observed at acidic $\mathrm{pH}$ values, with minimal signal between $\mathrm{pH} 5$ and 7 observed, before a dramatic increase is seen at $\mathrm{pH}$ $8{ }^{18}$ In contrast, $\mathrm{pH}$ analysis of scopolamine hydrobromide revealed that a measurable signal is observed across all $\mathrm{pH}$ values. This includes acidic $\mathrm{pH}$ values, with an increase in signal observed at both $\mathrm{pH} 6$ and $\mathrm{pH}$ 8. Despite the almost identical structure of the two alkaloids, their $\mathrm{p} K_{\mathrm{a}}$ values are different, with atropine reported at $9.85^{48}$ and scopolamine hydrobromide at 7.6. ${ }^{49}$ The difference in $\mathrm{p} K_{\mathrm{a}}$ and subsequently the proportion of free base species present at different $\mathrm{pH}$ values thus presents the opportunity to exploit this difference to facilitate the identification of the alkaloids separately within mixed samples. Scopolamine's lower $\mathrm{p} K_{\mathrm{a}}$ and hence lower basicity compared with atropine means that more of its nonpronated, and hence oxidizable form, will be present at lower $\mathrm{pH}$ values. In fact the proportion of free base scopolamine present at a physiological $\mathrm{pH}$ of 7.4 is reported to be 70 times more than atropine under the same conditions. ${ }^{38}$ Thus, it is therefore not surprising when directly comparing the ECL intensity dependence with $\mathrm{pH}$ for both species that there are obvious $\mathrm{pH}$ ranges where emission from one species can be essentially switched off. In contrast to ECL, there was no observable impact on the $\mathrm{CVs}$ due to $\mathrm{pH}$, see Figure S3. This is most likely due to the $\mathrm{Ru}^{2+/ 3+}$ species being the predominant feature in the $\mathrm{CV}$. The onset of ECL does correlate with the $\mathrm{CV}$ responses. It becomes clear when examining the ECL intensity dependence with $\mathrm{pH}$ for each species that acidic $\mathrm{pH}$ values offer the best compromise between negating the signal response from atropine and obtaining the maximum signal from scopolamine, see Figure 2. As such the optimum $\mathrm{pH}$ identified for the detection of scopolamine in the presence of atropine was determined to be $\mathrm{pH}$ 6. In contrast, scopolamine is observed to emit a considerable signal at all $\mathrm{pH}$ values where atropine emits. Although it could be thought that $\mathrm{pH} 11$ would offer a viable $\mathrm{pH}$ to optimize atropine emission and negate scopolamine emission, we have previously shown that emission at this $\mathrm{pH}$ is due to the catalyzed degradation of atropine under alkaline conditions, leading to the formation of tropine and tropic acid. ${ }^{18,50}$ As such, this degradation prevented us from quantifying atropine in the presence of scopolamine with an inability to accurately control the concentration of atropine still present at this $\mathrm{pH}$. Furthermore, tropine is also found to produce an ECL response and as such will contribute to the observed signal. Therefore, the proportion of the signal due to tropine compared with atropine could not be discerned. ${ }^{18}$

Analytical Performance. Prior to analysis of mixed samples, it was necessary to prepared suitable calibration curves at the chosen $\mathrm{pH}$ values. The optimum $\mathrm{pH}$ for the determination of scopolamine in the presence of atropine was chosen as $\mathrm{pH}$ 6. Although there are other $\mathrm{pH}$ values which 


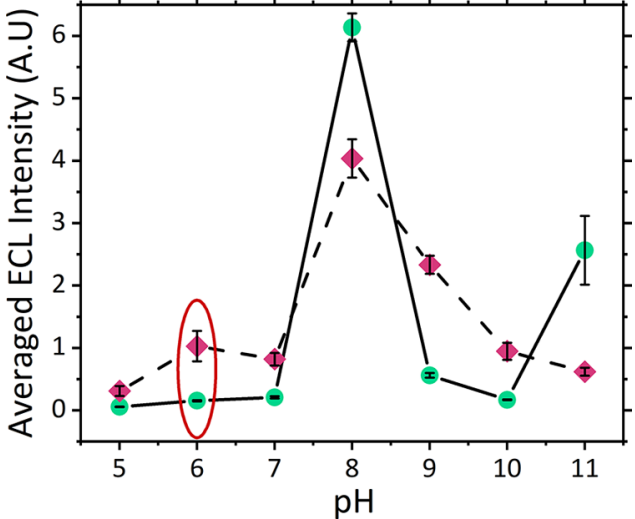

Figure 2. Comparison of ECL intensities with $\mathrm{pH}$ for atropine sulfate (green) and scopolamine hydrobromide (pink), with $\mathrm{pH} 6$ highlighted at the optimum $\mathrm{pH}$. Both species were prepared in $0.1 \mathrm{M}$ $\mathrm{LiClO}_{4}$ at the desired $\mathrm{pH}$. Measurements were collected at a scan rate of $100 \mathrm{mV} \mathrm{s}^{-1}$ across $0.5 \leq E \leq 1.36 \mathrm{~V}$ vs Ag at a PMT setting of 0.45 $\mathrm{V}$ for atropine sulfate and $0.6 \mathrm{~V}$ for scopolamine hydrobromide.

resulted in greater ECL intensities, $\mathrm{pH} 6$ produced the greatest difference in ECL response for the two alkaloids meaning it would be ideally suited to discriminate the presence of scopolamine over atropine. At other $\mathrm{pH}$ values, the contribution from atropine ECL would not make quantification of scopolamine possible. $\mathrm{pH} 10$ was also considered, but the atropine response was larger than that observed for $\mathrm{pH} 6$ where it was negligible, in addition to $\mathrm{pH} 10$ lying close to where alkaline degradation of atropine is observed.

Thus, a $\mathrm{pH} 6$ calibration curve was prepared for scopolamine hydrobromide. The developed $\left[\mathrm{Ru}(\mathrm{bpy})_{3}\right]^{2+}$ film SPE sensor utilized in this study has previously demonstrated satisfactory analytical performance, including reproducibility of $3.0 \%$ and repeatability of $9.2 \%$ and exhibits the same behavior here. ${ }^{18}$ The limit of detection (LOD) for each calibration was calculated as 0.418 and $0.440 \mu \mathrm{M}$ for $\mathrm{pH} 6$ and 8 , respectively, based upon the standard deviation of the $y$ intercept and slope of the regression line. ${ }^{51}$ The influence of scopolamine hydrobromide concentration on ECL intensity at pH 6 was investigated across a range of $0.625-250 \mu \mathrm{M}$. As analyte concentration increased the ECL intensity was observed to linearly increase in proportion with maximum ECL response between 0.625 and $100 \mu \mathrm{M}$ with a coefficient $R^{2}$ value of 0.999 , see the inset of Figure 3a. Above $100 \mu \mathrm{M}$, a plateau effect was observed (see Figure S4) which prevented quantification above this concentration. This plateau effect is not unsurprising and is a previously reported limitation of ECL. ${ }^{18,52}$ At higher analyte concentrations, the consumption of ruthenium(III) becomes the limiting step, where it is consumed at a rate quicker than it can be produced. As such, a point is reached when the ECL intensity will no longer increase as analyte concentration increases. ${ }^{18,52}$ To tackle this, the ruthenium concentration present could be increased within the film or a dilution factor taken into account. However, the concentrations of scopolamine and atropine are likely to be encountered in any "real-world" scenario would be far lower, with concentrations typically ranging from $\sim 0.99$ to $20 \mu \mathrm{M}$ in biological or herbal material samples; therefore, it was not currently necessary to concentrate on quantification of these higher concentrations. ${ }^{53-56}$ However, this does pose a sizable limitation in the current methodology, should it be required for quantification rather than qualification of higher concentration
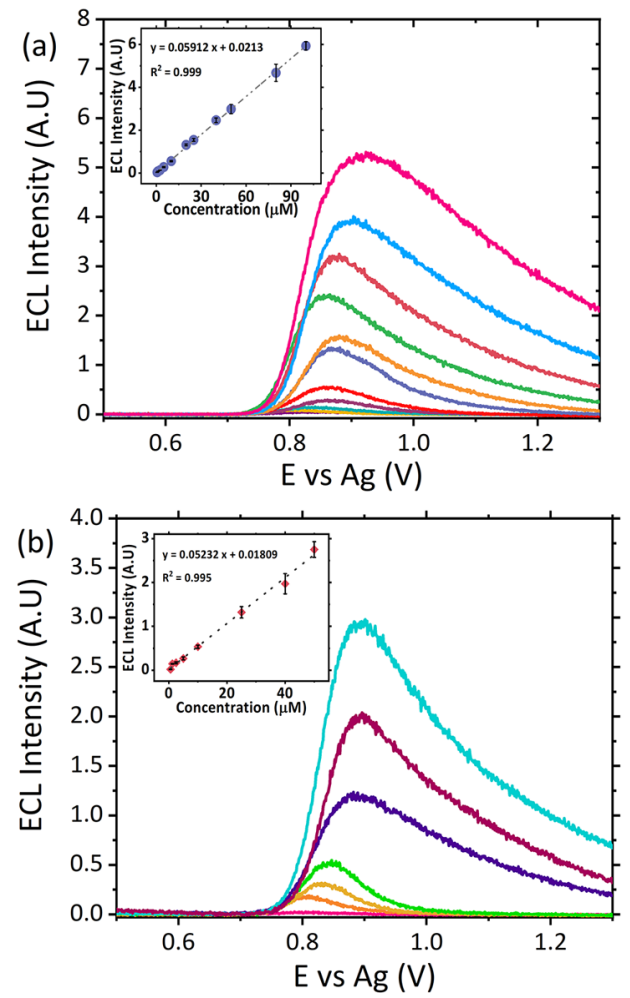

Figure 3. Dependence of ECL intensity with scopolamine hydrobromide concentration at (a) $\mathrm{pH} 6$ between 0.625 and $100 \mu \mathrm{M}$ and (b) $\mathrm{pH} 8$ between 0.625 and $50 \mu \mathrm{M}$. All samples were prepared in the appropriate $\mathrm{pH} 0.1 \mathrm{M} \mathrm{LiClO}_{4}$ and measured at a scan rate of $100 \mathrm{mV}$ $\mathrm{s}^{-1}$ across $0.5 \leq E \leq 1.26 \mathrm{~V}$ vs Ag at a PMT setting of $0.6 \mathrm{~V}$. Insets show the trend of maximum ECL intensity against scopolamine hydrobromide concentration.

samples, and as such, development to improve the applicable concentration range is ongoing.

In addition to $\mathrm{pH} 6$, a calibration curve was also prepared at $\mathrm{pH} 8$, the optimum $\mathrm{pH}$ where maximum ECL intensity is observed for each analyte. The same concentration range as for $\mathrm{pH} 6$ was investigated, with a linearly proportional response observed with scopolamine concentration at a maximum ECL intensity with a coefficient $R^{2}$ value of 0.995 , see Figure $3 b$, inset. In contrast to $\mathrm{pH} 6$, at $\mathrm{pH} 8$ the linear range was only observed between 0.625 and $50 \mu \mathrm{M}$, see Figure $3 \mathrm{~b}$. Above 50 $\mu \mathrm{M}$, we began to observe the previously described plateau effect (refer to Figure S5). At concentrations above $80 \mu \mathrm{M}$ scopolamine at $\mathrm{pH} 8$, a decrease in the ECL intensity can be observed. This may partly be due to the more easily oxidizable form at this $\mathrm{pH}$ undergoing direct oxidation generating the electro-N-dealkylation speciese (aposcopolamine). The formation of aposcopolamine would result in a drop in concentration of the ECL coreactant, scopolamine, and therefore result in a drop in ECL production. This can be attributed to a far greater proportion of scopolamine being present in its nonprotonated oxidizable form at this $\mathrm{pH}$. Thus, ruthenium(III) becomes the limiting reagent at a much lower total concentration due to a higher proportion of scopolamine species present, which are able to undergo ECL. As can be seen in both Figure $3 \mathrm{a}, \mathrm{b}$, in addition to ECL intensity increasing with scopolamine concentration, we also observe a slight shift in the potential at which the maximum response is observed. This effect is not unexpected and is consistent with prior 
Table 1. Summary of Atropine Sulfate Concentrations Added and Scopolamine Hydrobromide Concentrations Calculated at Each pH

$\begin{array}{cccc}\mathrm{pH} & \text { [atropine] }(\mu \mathrm{M}) & \text { average calculated [scopolamine] }(\mu \mathrm{M}) \\ 6 & 12.5 & 50 & 49.7 \\ 6 & 25 & 50 & 50.8 \\ 6 & 50 & 50 & 49.3 \\ 6 & 100 & 50 & 50.3 \\ 8 & 12.5 & 50 & 55.1 \\ 8 & 25 & 50 & 67.9 \\ 8 & 50 & 50 & 90.2 \\ 8 & 100 & 50 & 54.5\end{array}$

literature where a shift is observed at both ruthenium modified carbon electrodes and unmodified graphite electrodes. ${ }^{18,57}$ This shift can likely be attributed to two effects. As the concentration of scopolamine is increased and we approach the concentrations at which ruthenium(III) becomes the limiting reagent, it becomes more difficult to produce the oxidized species required to undergo ECL. Moreover, as more scopolamine is present and undergoes the irreversible ECL mechanisms, a greater concentration of the byproducts, scopine and formaldehyde, are produced leading to a shift in the $\mathrm{pH}$ of the sample within the inner Helmholtz plane close to the electrode surface. ${ }^{18,52,57}$ However, at $\mathrm{pH} 6$, no significant response from atropine was observed so this would not impact on the ability to determine the presence of scopolamine. At $\mathrm{pH}$ 8 , the earlier onset of ECL production would indicate the presence of atropine. Therefore, it is necessary to have both results to identify the presence of both alkaloids. Without both sets of data, an indication of the total alkaloid presence could be taken but identification of the number of alkaloids presence would not be possible.

Mixed Alkaloid Sample Analysis. To establish whether the analyte $\mathrm{p} K_{\mathrm{a}}$ could be exploited to successfully quantify individual analytes with similar ECL emission mechanisms within the same sample matrix, the two tropane alkaloids atropine and scopolamine were used as model compounds in this proof-of-concept study. Samples containing both alkaloids were prepared and measured at $\mathrm{pH} 6$, where only scopolamine emission is expected, $\mathrm{pH} 8$ where both species produce the greatest ECL intensity, and $\mathrm{pH} 11$ where atropine's emission over scopolamine is the greatest. The scopolamine concentration was kept constant in both sets of $\mathrm{pH} 6$ and 8 samples at $50 \mu \mathrm{M}$ while the atropine sulfate concentration was varied between 12.5 and $100 \mu \mathrm{M}$. For $\mathrm{pH} \mathrm{11}$, concentrations were reversed, with atropine maintained at $50 \mu \mathrm{M}$ and scopolamine varied between 12.5 and $100 \mu \mathrm{M}$. Assessment on the ECL signal intensity due to the presence of atropine was made by comparing the calculated concentrations of scopolamine hydrobromide at both $\mathrm{pH} 6$ and 8 to the known concentrations added. Scopolamine hydrobromide concentrations were calculated using the relevant calibration curves constructed for each $\mathrm{pH}$, see Figure 3a,b, the results of which are summarized in Table 1 . In order to establish whether a sample contained only scopolamine or if another alkaloid was present, in this case atropine it is necessary to have the information for the expected scopolamine signal both at $\mathrm{pH} 6$ and 8 of pure standards. These can then be used as system standards, in a similar manner as is performed for more traditional analytical techniques such as HPLC.

The ECL intensity of the $\mathrm{pH} 8$ samples demonstrated a trend of increasing ECL intensity as the atropine concentration increased between 12.5 and $50 \mu \mathrm{M}$. After this, a decrease in overall ECL intensity was observed, see Figure 4a. This decrease is not unexpected and can be attributed to the ruthenium(III) species becoming the rate limiting reagent. At $\mathrm{pH} 8$, the free base or nonpronated form of both species will be the dominate form present. Previously, we have discussed how $50 \mu \mathrm{M}$ scopolamine hydrobromide was the maximum concentration which produced an increasing ECL intensity with increased concentration. Above $50 \mu \mathrm{M}$, the ECL intensity was no longer dependent on the scopolamine concentration, and thus $50 \mu \mathrm{M}$ was the maximum concentration which could be accurately quantified. Thus, with scopolamine hydrobromide at its maximum quantifiable concentration and atropine sulfate also present at its maximum linearly dependent concentration of $100 \mu \mathrm{M}$, it is thus not unsurprising that a decrease in ECL intensity is observed. As can be seen in Table 1 , the concentration of scopolamine calculated within the $\mathrm{pH} 8$ mixed samples is far greater than the $50 \mu \mathrm{M}$ present. Furthermore, examination of the ECL signals, such as those shown in Figure $4 \mathrm{~b}$, demonstrate that the total ECL signal observed for the mixed alkaloid sample is not the result of direct addition of the two individual signals for atropine and scopolamine at this $\mathrm{pH}$. As such, the ECL intensity of scopolamine hydrobromide cannot be corrected by direct subtraction of the atropine signal.

In contrast, analysis at $\mathrm{pH} 6$ displayed no increase in total ECL intensity despite the increasing concentrations of atropine sulfate present, see Figure 4c. Furthermore, examination of the signals obtained for $50 \mu \mathrm{M}$ scopolamine are almost identical to those observed for the mixed samples, see Figure $4 \mathrm{~d}$. Table 1 contains a summary of the scopolamine concentrations calculated at pH 6 and demonstrates that, despite the presence of increasing concentrations of atropine, the calculated scopolamine concentrations remain constant at approximately $50 \mu \mathrm{M}$, hence, demonstrating a good accuracy of the methodology. This promising result demonstrated that by exploiting the difference in $\mathrm{p} K_{\mathrm{a}}$ values, compounds with similar chemical structures and hence ECL emission mechanisms can be successfully quantified within a mixed sample where more than one species produces an ECL response.

As previously discussed, when samples at $\mathrm{pH} 11$ were analyzed, the base catalyzed degradation of atropine to the ECL active degradation product, tropine, prevented quantification at this $\mathrm{pH}$. However, with scopolamine producing a minimal emission intensity of $\sim 0.34$ A.U at $\mathrm{pH} 11$, we are able to confidently identify the presence of a secondary species; in this case, atropine is producing the observed signal increase, refer to Figure 5. Due to the significant increase in signal intensity when atropine is present in addition to scopolamine at $\mathrm{pH} 11$, we can adequately use $\mathrm{pH}$ controlled ECL as a 

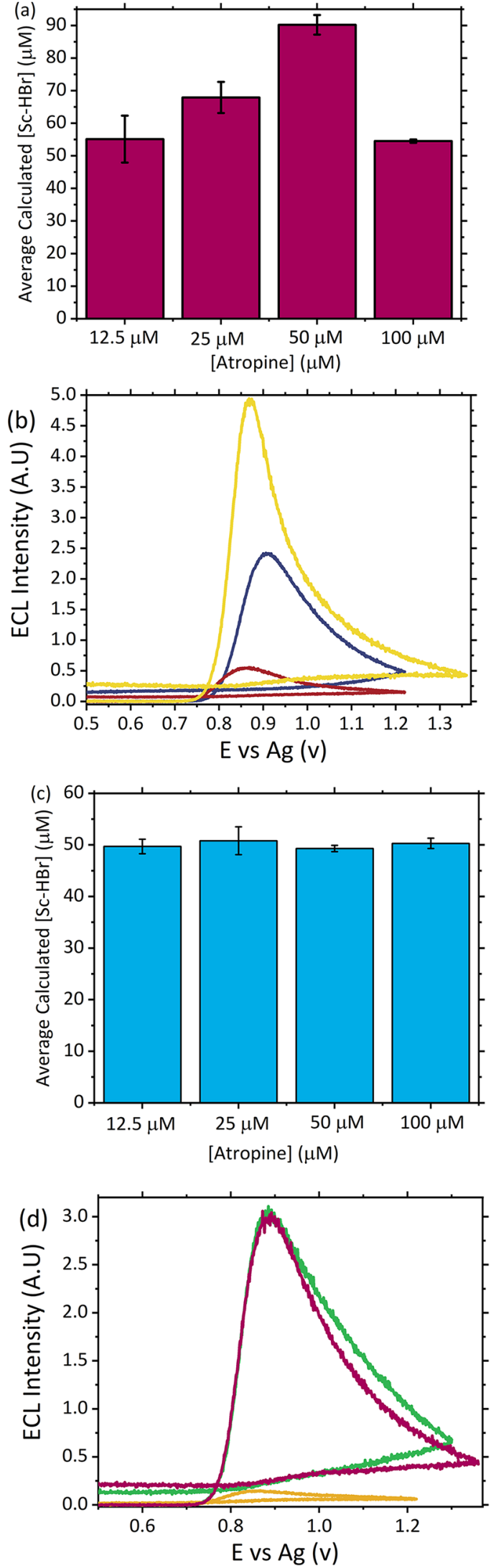

Figure 4. (a) Calculated scopolamine hydrobromide ( $\mathrm{Sc}-\mathrm{Hr}$ ) concentrations in the presence of varying concentrations of atropine sulfate at $\mathrm{pH}$ 8. Concentrations calculated based upon maximum ECL intensity. (b) ECL responses of $50 \mu \mathrm{M}$ atropine sulfate (pink), $50 \mu \mathrm{M}$ scopolamine hydrobromide (purple), and $50 \mu \mathrm{M}$ atropine sulfate and
Figure 4. continued

scopolamine hydrobromide (yellow) in $\mathrm{pH} 80.1 \mathrm{M} \mathrm{LiClO}_{4}$ as the electrolyte and measured at a scan rate of $100 \mathrm{mV} \mathrm{s}^{-1}$ across $0.5 \leq E$ $\leq 1.36 \mathrm{~V}$ vs Ag at a PMT setting of $0.6 \mathrm{~V}$. (c) Calculated scopolamine hydrobromide concentrations in the presence of varying concentrations of atropine sulfate at $\mathrm{pH}$ 6. Concentrations calculated based upon maximum ECL intensity. (d) ECL responses of $50 \mu \mathrm{M}$ atropine sulfate (yellow), $50 \mu \mathrm{M}$ scopolamine hydrobromide (green), and 50 $\mu \mathrm{M}$ atropine sulfate and scopolamine hydrobromide (pink) in $\mathrm{pH} 6$ $0.1 \mathrm{M} \mathrm{LiClO}_{4}$ as the electrolyte and measured at a scan rate of 100 $\mathrm{mV} \mathrm{s}^{-1}$ across $0.5 \leq E \leq 1.36 \mathrm{~V}$ vs Ag at a PMT setting of $0.6 \mathrm{~V}$.

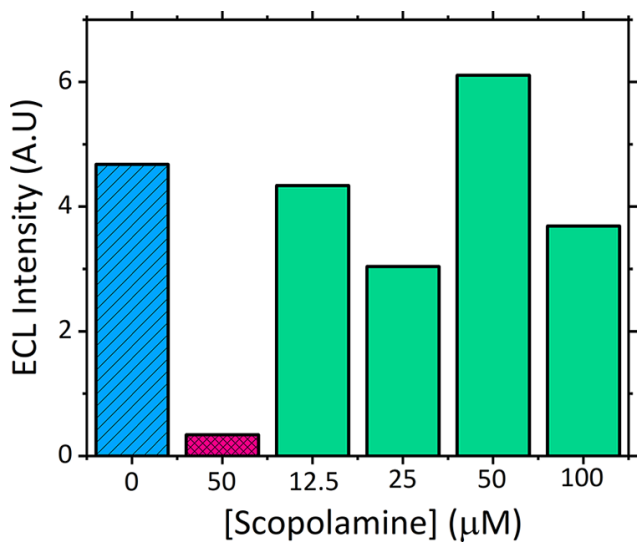

Figure 5. ECL intensity with varying concentrations of atropine sulfate in the presence of $50 \mu \mathrm{M}$ scopolamine hydrobromide at $\mathrm{pH} 11$ (green). The pink bar represents the ECL intensity observed $50 \mu \mathrm{M}$ scopolamine hydrobromide only at $\mathrm{pH} 11$. The blue bar represents the ECL intensity observed for $50 \mu \mathrm{M}$ atropine sulfate only at $\mathrm{pH} 11$.

screening protocol to identify the number of species present in a potential mixed sample. Moreover, although in this current contribution we have not been able to quantify the multiple species present, we have established a future application for chemometric modeling with ECL for the first time. By analyzing samples at $\mathrm{pH} 6$ and subsequently $\mathrm{pH} 11$, one can identify if both tropane alkaloids are present. Using $\mathrm{pH} 6$ to negate emission from atropine, the concentration of scopolamine can be confidently determined. It is then probable that knowing the concentration of scopolamine that a calibration model could be constructed incorporating this known concentration. Chemometric modeling would then facilitate the removal of the signal contribution from scopolamine, thus allowing for the determination of the proportion of the signal attributed solely to atropine. Using this model, it would then be possible to determine the concentration of atropine present in the unknown sample.

\section{CONCLUSION}

In this work, we have demonstrated for the first time the ability to identify between almost identical chemical structures through a basic $\left[\mathrm{Ru}(\mathrm{bpy})_{3}\right]^{2+}$ based ECL sensor. Prior to this, species similar in structure, such as the two alkaloids used here, could not be differentiated from one another via ECL without employing either separation strategies prior to detection or complex sample preparation to physically separate out individual components of a mixture. ${ }^{20,58} \mathrm{ECL}$ has long suffered from limited specificity as a result of its broad emission spectra leading to indistinctive peaks with groups of compounds such as amines, all producing emission in the same 
potential region. Previously, we have reported on the use of the same basic $\left[\mathrm{Ru}(\mathrm{bpy})_{3}\right]^{2+}$ sensor for the detection of atropine within herbal plant material and highlighted the inability to distinguish whether atropine, scopolamine, or both were responsible for the observed signal. ${ }^{18}$ By utilizing $\mathrm{pH}$ and exploiting the differences in $\mathrm{p} K_{a}$, we have been able to develop a methodology which can be used to qualitatively determine whether a single species or multiple are responsible for the observed signal. Furthermore, although we have not been able to quantify both tropane alkaloids at this time, our $\mathrm{pH}$ controlled ECL strategy can be successfully used for the quantification of scopolamine within a mixed sample containing various concentrations of atropine. Both tropane alkaloids as mentioned above have been previously shown to emit within the same potential region and thus are known to cause interference for qualifying or quantifying the alkaloids individually, when they are within the same matrix. Here we have shown that by closely studying the impact of $\mathrm{pH}$ on ECL emission, we can identify a region where a single species' emission can in essence be switched off facilitating the qualification or quantification of the remaining emitting species. Although this is a promising step forward to increase the specificity offered by ECL, further development is still required. Although here we have been able to accurately quantify scopolamine in the presence of atropine, we could not successfully perform the reverse. It therefore stands that the same problem would be encountered for other compound groups. However, it is hoped that by applying chemometric modeling to overcome this limitation will allow us to quantify more species in future. Currently we have solely focused on the tropane alkaloid family, and thus it is vital that other groups of structurally similar compounds are also investigated in the same manner to understand the wider applicability of the $\mathrm{pH}$ controlled ECL. While the initial studies reported within are promising and despite ECL holding a number of advantages in comparison to its rivals, its limited specificity is currently hindering applications of the technique among the wider analytical community. As such, alternative methods to further improve its specificity are imperative if ECL is to become a more widely employed technique.

\section{ASSOCIATED CONTENT}

\section{S Supporting Information}

The Supporting Information is available free of charge at https://pubs.acs.org/doi/10.1021/acs.analchem.9b04922.

$\mathrm{CV}$ responses of $2.5 \mathrm{mM}$ atropine sulfate, $2.5 \mathrm{mM}$

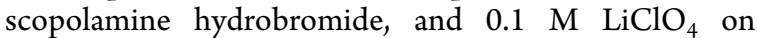
unmodified screen-printed electrodes (Figure S1), CV responses of $50 \mu \mathrm{M}$ atropine sulfate, $50 \mu \mathrm{M}$ scopolamine hydrobromide, and $0.1 \mathrm{M} \mathrm{LiClO}_{4}$ (Figure S2), CV responses of atropine sulfate and scopolamine hydrobromide at $\mathrm{pH} 6$ and $\mathrm{pH} 8$ (Figure S3), maximum ECL responses for scopolamine hydrobromide between 50 and $250 \mu \mathrm{M}$ at $\mathrm{pH} 6$ (Figure S4), and maximum ECL responses for scopolamine hydrobromide between 50 and $200 \mu \mathrm{M}$ at $\mathrm{pH} 8$ (Figure S5) (PDF)

\section{AUTHOR INFORMATION}

\section{Corresponding Author}

*E-mail: lynn.dennany@strath.ac.uk.

ORCID 1

Lynn Dennany: 0000-0002-5481-1066

\section{Author Contributions}

The manuscript was written through contributions of all authors.

\section{Notes}

The authors declare no competing financial interest.

All data underpinning this publication are openly available from the University of Strathclyde KnowledgeBase at 10. 15129/69cd9d07-ac4a-437b-8d06-70d19a0bb2b3

\section{ACKNOWLEDGMENTS}

The authors would like to acknowledge The Carnegie Trust for the Universities of Scotland for the Ph.D. scholarship funding which has supported this work.

\section{REFERENCES}

(1) Shaw, L.; Dennany, L. Current Opinion in Electrochemistry 2017, $3,23-28$.

(2) de Araujo, W. R.; Cardoso, T. M. G.; da Rocha, R. G.; Santana, M. H. P.; Muñoz, R. A. A.; Richter, E. M.; Paixão, T. R. L. C.; Coltro, W. K. T. Anal. Chim. Acta 2018, 1034, 1-21.

(3) Chemat, F.; Garrigues, S.; de la Guardia, M. Curr. Opin. Green Sustain. Chem. 2019, 19, 94

(4) Bard, A. J.; Faulkner, L. R.; Leddy, J.; Zoski, C. G. In Electrochemical Methods: Fundamentals and Applications; Wiley New York: New York, 1980; pp 736-766.

(5) Richter, M. M. Chem. Rev. 2004, 104, 3003-3036.

(6) Miao, W. Chem. Rev. 2008, 108, 2506-2553.

(7) Truong, C. K. P.; Nguyen, T. D. D.; Shin, I.-S. BioChip J. 2019, 13, 203-216

(8) O’Reilly, E. J.; Conroy, P. J.; Hearty, S.; Keyes, T. E.; O'Kennedy, R.; Forster, R. J.; Dennany, L. RSC Adv. 2015, 5, 6787467877

(9) Delaney, J. L.; Doeven, E. H.; Harsant, A. J.; Hogan, C. F. Anal. Chim. Acta 2013, 790, 56-60.

(10) Doeven, E. H.; Barbante, G. J.; Harsant, A. J.; Donnelly, P. S.; Connell, T. U.; Hogan, C. F.; Francis, P. S. Sens. Actuators, B 2015, 216, 608-613.

(11) Dennany, L.; O’Reilly, E. J.; Keyes, T. E.; Forster, R. J. Electrochem. Commun. 2006, 8, 1588-1594.

(12) Pastore, P.; Badocco, D.; Zanon, F. Electrochim. Acta 2006, 51, 5394-5401.

(13) An, D.; Chen, Z.; Zheng, J.; Chen, S.; Wang, L.; Huang, Z.; Weng, L. Food Chem. 2015, 168, 1-6.

(14) McGeehan, J.; Dennany, L. Forensic Sci. Int. 2016, 264, 1-6.

(15) Stewart, A. J.; Brown, K.; Dennany, L. Anal. Chem. 2018, 90, 12944-12950.

(16) Yang, X.-Y.; Xu, C.-Y.; Yuan, B.-Q.; You, T.-Y. Chin. J. Anal. Chem. 2011, 39, 1233-1237.

(17) Zhang, A.; Miao, C.; Shi, H.; Xiang, H.; Huang, C.; Jia, N. Sens. Actuators, B 2016, 222, 433-439.

(18) Brown, K.; McMenemy, M.; Palmer, M.; Baker, M. J.; Robinson, D. W.; Allan, P.; Dennany, L. Anal. Chem. 2019, 91, 12369-12376.

(19) Cao, W.; Liu, J.; Qiu, H.; Yang, X.; Wang, E. Electroanalysis 2002, 14, 1571-1576.

(20) Gao, Y.; Tian, Y.; Wang, E. Anal. Chim. Acta 2005, 545, 137141.

(21) Li, J.; Chun, Y.; Ju, H. Electroanalysis 2007, 19, 1569-1574.

(22) Yuan, B.; Zheng, C.; Teng, H.; You, T. J. Chromatogr.A 2010, $1217,171-174$

(23) Bozokalfa, G.; Akbulut, H.; Demir, B.; Guler, E.; Gumus, Z. P.; Odaci Demirkol, D.; Aldemir, E.; Yamada, S.; Endo, T.; Coskunol, H.; Timur, S.; Yagci, Y. Anal. Chem. 2016, 88, 4161-4167.

(24) Oueslati, R.; Cheng, C.; Wu, J.; Chen, J. Biosens. Bioelectron. 2018, 108, 103-108.

(25) Gao, J.; Chen, Z.; Mao, L.; Zhang, W.; Wen, W.; Zhang, X.; Wang, S. Talanta 2019, 199, 178-183. 
(26) Zhou, J.; Gan, N.; Hu, F.; Li, T.; Zhou, H.; Li, X.; Zheng, L. Sens. Actuators, B 2013, 186, 300-307.

(27) de Oliveira, L. S.; Balbino, M. A.; de Menezes, M. M. T.;

Dockal, E. R.; de Oliveira, M. F. Microchem. J. 2013, 110, 374-378.

(28) de Oliveira, L.; dos Santos Poles, A.; Balbino, M.; Teles de Menezes, M.; de Andrade, J.; Dockal, E.; Tristão, H.; de Oliveira, M. Sensors 2013, 13, 7668.

(29) Asturias-Arribas, L.; Alonso-Lomillo, M. A.; DomínguezRenedo, O.; Arcos-Martínez, M. J. Anal. Chim. Acta 2014, 834, 3036.

(30) Xie, H.; Li, X.; Zhao, L.; Han, L.; Zhao, W.; Chen, X. Sens. Actuators, B 2016, 222, 226-231.

(31) Stewart, A. J.; Hendry, J.; Dennany, L. Anal. Chem. 2015, 87, 11847-11853.

(32) Kim, H. J.; Lee, K.-S.; Jeon, Y.-J.; Shin, I.-S.; Hong, J.-I. Biosens. Bioelectron. 2017, 91, 497-503.

(33) Li, H.; Sedgwick, A. C.; Li, M.; Blackburn, R. A. R.; Bull, S. D.; Arbault, S.; James, T. D.; Sojic, N. Chem. Commun. 2016, 52, 1284512848.

(34) Yang, X.-Y.; Xu, C.-Y.; Yuan, B.-Q.; You, T.-Y. Chin. J. Anal. Chem. 2011, 39, 1233-1237.

(35) Florea, A.; Schram, J.; de Jong, M.; Eliaerts, J.; Van Durme, F.; Kaur, B.; Samyn, N.; De Wael, K. Anal. Chem. 2019, 91, 7920-7928.

(36) Rudyk, R.; Checa, M.; Guzzetti, K.; Iramain, M.; Brandán, S. Int. J. Soc. Res. Methodol 2018, 10, 70-97.

(37) Márquez, M. J.; Iramain, M. A.; Brandán, S. A. Int. J. Soc. Res. Methodol. 2018, 12, 97-140.

(38) Weinstein, H.; Srebrenik, S.; Maayani, S.; Sokolovsky, M. J. Theor. Biol. 1977, 64, 295-309.

(39) He, L.; Cox, K. A.; Danielson, N. D. Anal. Lett. 1990, 23, 195210.

(40) Sastri, V. S. In Underground Pipeline Corrosion; Orazem, M. E., Ed.; Woodhead Publishing, 2014; pp 166-211.

(41) Miao, W.; Choi, J.-P.; Bard, A. J. J. Am. Chem. Soc. 2002, 124, 14478-14485.

(42) Sentic, M.; Milutinovic, M.; Kanoufi, F.; Manojlovic, D.; Arbault, S.; Sojic, N. Chem. Sci. 2014, 5, 2568-2572.

(43) Portis, L. C.; Bhat, V. V.; Mann, C. K. J. Org. Chem. 1970, 35, 2175-2178.

(44) Laube, B. L.; Asirvatham, M. R.; Mann, C. K. J. Org. Chem. 1977, 42, 670-674.

(45) Dennany, L.; O’Reilly, E. J.; Innis, P. C.; Wallace, G. G.; Forster, R. J. Electrochim. Acta 2008, 53, 4599-4605.

(46) Zhang, A.; Miao, C.; Shi, H.; Xiang, H.; Huang, C.; Jia, N. Sens. Actuators, B 2016, 222, 433-439.

(47) Xiang, Q.; Yang, X. D.; Gao, Y. Adv. Mater. Res. 2014, 989994, 1007-1010.

(48) Dettbarn, W. D.; Heilbronn, E.; Hoskin, F. C. G.; Kitz, R. Neuropharmacology 1972, 11, 727-732.

(49) Muhtadi, F. J.; Hassan, M. M. A. In Analytical Profiles of Drug Substances; Florey, K., Ed.; Academic Press, 1990; pp 477-551.

(50) Zvirblis, P.; Socholitsky, I.; Kondritzer, A. A. J. Am. Pharm. Assoc., Sci. Ed. 1956, 45, 450-454.

(51) Shrivastava, A.; Gupta, V. Chron. Young Sci. 2011, 2, 21-25.

(52) Oja, S. M.; Zhang, B. ChemElectroChem 2016, 3, 457-464.

(53) Winek, C. L.; Wahba, W. W.; Winek, C. L.; Balzer, T. W. Forensic Sci. Int. 2001, 122, 107-123.

(54) Boros, B.; Farkas, Á.; Jakabová, S.; Bacskay, I.; Kilár, F.; Felinger, A. Chromatographia 2010, 71, 43-49.

(55) Le Garff, E.; Delannoy, Y.; Mesli, V.; Hédouin, V.; Tournel, G. Forensic Sci. Int. 2016, 261, e17-e21.

(56) Lusthof, K. J.; Bosman, I. J.; Kubat, B.; Vincenten-van Maanen, M. J. Forensic Sci. Int. 2017, 274, 79-82.

(57) Shen, L.-H.; Wang, H.-N.; Chen, P.-J.; Yu, C.-X.; Liang, Y.-D.; Zhang, C.-X. J. Food Drug Anal. 2016, 24, 199-205.

(58) Dar, R. A.; Brahman, P. K.; Tiwari, S.; Pitre, K. S. Colloids Surf., B 2012, 91, 10-17. 